\title{
COMPUTER BASED ASSESSMENT OF GENERAL MOVEMENTS IN YOUNG INFANTS USING ONE OR TWO VIDEO RECORDINGS
}

\author{
L. Adde ${ }^{1,2}$, M. Langaas ${ }^{3}$, A.R. Jensenius ${ }^{4}$, J.L. Helbostad ${ }^{5,6}$, R. Støen ${ }^{1,7}$ \\ ${ }^{1}$ Department of Laboratory Medicine, Children's and Women's Health, Norwegian University of Science and \\ Technology, ${ }^{2}$ Department of Clinical Services, St. Olavs Hospital, Trondheim University Hospital, \\ ${ }^{3}$ Department of Mathematical Sciences, Norwegian University of Science and Technology, Trondheim, \\ ${ }^{4}$ Department of Musicology, University of Oslo, Oslo, ${ }^{5}$ Department of Neuroscience, Norwegian University \\ of Science and Technology, ${ }^{6}$ St. Olav Hospital, Trondheim University Hospital, ${ }^{7}$ Department of Pediatrics, \\ St. Olav Hospital, Trondheim University Hospital, Trondheim, Norway
}

Background and aims: Absent fidgety movements (FMs) in infants is a strong marker for cerebral palsy. A computer based video analysis tool (General Movement Toolbox, GMT) has been developed to identify infants with absent FMs. Our aim was to evaluate whether a mean of two recordings was superior to one recording in computer based FM classification.

Methods: Fifty-two term and preterm infants ( 24 boys, 28 girls, 26 preterm; median preterm gestational age $30 \mathrm{wks}$, range 23-36 wks) were recruited. All infants had two video recordings between 9 and 17 weeks post term age, and FMs were classified based on the Prechtl approach of general movement assessment (GMA). The GMT variable reflecting variability of the centre of the movement $\left(\mathrm{C}_{\mathrm{SD}}\right)$ was used for quantitative FM classification. Areas under receiver operating characteristic curves were used as a measure of strength of the classification model. Logistic regression with leave-one-out cross validation was used.

Results: Median ages at first and second recordings were 11 and 15 weeks post term, respectively. Eighteen of 104 recordings were classified with absent FMs by GMA. When using the computer generated variable $\mathrm{C}_{\mathrm{SD}}$, area under curve was 0.82 using the first and 0.81 using the second recording. When using the mean value of $\mathrm{C}_{\mathrm{SD}}$ from both recordings, area under curve increased to 0.88 .

Conclusions: Computer based video analysis estimating the variability of the centre of movement $\left(\mathrm{C}_{\mathrm{SD}}\right)$ can be used to classify FMs. A more accurate model is provided by averaging values from two recordings compared to a single recording. 